\title{
The quality of care in family planning: a case study of Chogoria, Eastern Kenya
}

\author{
Evasius KABURU BAUNI
}

\section{ABSTRACT}

This paper compares the quality of family planning services in the catchment areas of Chogoria and Maua hospitals, both in the Meru district of Kenya. The quality issues compared are personnel, types of methods, information, recruiting and counselling of clients, knowledge and source of modern contraceptives, desired family size, use of contraceptives, and satisfaction of providers and clients. A comparative approach is adopted to study the aspects of family planning that have made Chogoria relatively more successful than Maua and the rest of Kenya. The data used in this analysis is qualitative and was collected through personal observation, interviews, and group discussions with health care providers. The results show that while the family planning programmes of Chogoria and Maua are comparable in many respects, there are also important differences. For example, Chogoria family planning personnel were more knowledgeable about contraceptives and were more satisfied with the training provided by Chogoria hospital than were their counterparts in Maua. The relationship between the senior and the junior staff was more cordial in Chogoria. The content of the information about contraceptives provided to women attending clinics was similar in both hospitals. But the teachers in Chogoria were more knowledgeable and confident than those in Maua. In Chogoria, the decision to use family planning is jointly taken by the husband and the wife, and if a client fails to turn up for an appointment a follow-up is scheduled. In Maua, the decision to use family planning is taken unilaterally by the wife and defaulters are not followed up. The study showed that 78 percent and 33 percent of the participants were using modern family planning methods in Chogoria and Maua respectively. Three conclusions are drawn from the study. First, that the satisfaction of family planning providers and their clients contributes positively towards more knowledge and use of modern contraceptives. Second, that women feel more secure and comfortable with the methods they use if their husbands are involved in deciding whether or not to adopt them. Finally, follow-up services for those who fail to attend appointments helps to strengthen rapport between providers and clients and provides an opportunity to learn of the circumstances that lead to discontinuing the use of contraceptives.

\section{INTRODUCTION}

This paper compares the quality of family planning services in Chogoria and Maua, Kenya. The quality issues compared are: personnel, information, types of methods, recruiting and counselling of clients, knowledge and source of modern contraceptives, and satisfaction of providers and clients. These are not exhaustive, but they broadly cover the preparation, service delivery, and outcome of family planning, as described by Bruce (1990;2I,2:6I-9I). Compared to most other parts of Kenya, Chogoria has a relatively high prevalence of modern contraceptive use. In 1985, about 35 percent of Chogoria women of reproductive age were using modern contraception (Bauni, 1990:133), as compared to about 15 percent of women in Kenya as a whole (Kenya, 1989:35). The findings suggest that good quality services attract and retain many users of modern contraceptives.

\section{BACKGROUND INFORMATION}

The two areas being compared are the catchments of Chogoria and Maua hospitals, both in Meru district, Kenya. Both are under the management of Protestant churches. Chogoria hospital began in 1922 and is managed by the Presbyterian Church of East Africa (PCEA); Maua began in 1930 and is managed by the Methodist Church of Kenya.

Chogoria is located on the eastern slopes of Mount Kenya and Maua is on the slopes of the Nyambene hills. The two areas have similar ecological zones with warm, wet climates and rich volcanic soils. Similar cash crops (coffee, tea) and 
subsistence crops (maize, beans, bananas) are grown.

Each hospital provides both curative and preventive health services. The curative services treat ailments ranging from high-altitude respiratory diseases to low-altitude malaria. Preventive services, on the other hand, range from health education to the provision of maternal and child health $(\mathrm{MCH})$ and family planning. This paper assesses only the quality of family planning care in Chogoria and Maua. A comparative approach is adopted to learn which aspects of family planning have made Chogoria relatively more successful than Maua and the rest of Kenya.

\section{DATA AND METHODOLOGY}

The data used in this analysis are qualitative in nature and were collected by interviewing health care providers, participating in group discussions, attending meetings where information regarding family planning was disseminated, and observing the process of providing family planning services.

An interview with a nurse providing family planning information and services was carried out at each hospital. The interviews covered the types of family planning services offered by the hospital; how clients are recruited and sustained; and the problems, solutions, and future prospects of family planning. Visits were conducted to 8 clinics out of 36 in Chogoria and 5 out of 10 in Maua. During each visit, the researcher interacted with health educators, volunteers, health committees, traditional birth attendants, and family-planning clients. In addition, health workers were observed as they provided family planning information and services.

Finally, the researcher visited 60 households in the Chogoria catchment and 30 in the Maua catchment. During these household visits the researcher observed and took notes on the socioeconomic well-being of the home, discussed health and family planning issues with parents, and recruited the latter to participate in focus-group discussions. Participants in the group discussions were males and females between 20 and 40 years of age, having primary or secondary education, married or single, but with at least one child. The content of the group discussion included the role of children, family size, spacing of children, and family planning. For purposes of this paper, only the information on family planning is used.

\section{Limitations of the Study}

This study is qualitative and involves small samples which are not statistically representative of all the women in the catchment of Chogoria and Maua. The conclusions and the use of figures are therefore substantively limited to those who participated in the study. The quality of care issues discussed in this paper are not exhaustive but are only those which stood out when a general study of reproductive behaviour was being undertaken.

\section{Personnel and Types of Contraceptive Method}

In both hospitals the community health departments (CHD) are mainly responsible for organising and coordinating all primary health activities. These are comprised of health education, local disease control, essential drug supply, maternal child health, immunisation, treating minor ailments, nutrition, and family planning. These services are provided both by trained and qualified staff and by trained volunteers, traditional birth attendants (TBAs), field educators, and area health committees $(A H C)$. The services are offered in both the hospital clinics and the mobile and static clinics. A mobile clinic does not provide services on a daily basis while a static clinic does. About seven family planning methods are provided. These include birth-control pills, IUDs, injections, tubal ligations, vasectomies, condoms, and foaming tablets.

\section{Information}

The content of the information provided was similar in many respects in both hospitals. It was divided into two parts, one dealing with general health and hygiene, and the other with family planning. The need to use latrines, to wash regularly, to immunise children, and to attend antenatal clinics was emphasized. The teaching of family planning began by contrasting the benefits of a small family to the difficulties of a large one. Then the various methods of contraceptives were described (names and what they look like), 
followed by information on how the various methods are used and where to get them. Unfortunately, information on how the methods work and their various shortcomings were not adequately discussed.

In Chogoria, many women use family planning, but the majority are mothers aged between 25 and 35 years of age and having between two and four children. These mothers, the nurse claimed, "have understood the difficulties of modern life with many children more clearly than the older mothers, and have resolved to do something positive about it." In both Chogoria and Maua, women who have not given birth and are not about to get married have no access either to family planning information or to services.

\section{Recruiting and Counselling Clients for Family Planning}

In both Chogoria and Maua, mothers are told about family planning during antenatal and postnatal clinic visits. In addition, field educators inform the public about family planning during home visits and at gatherings such as a chief's baraza or a church event.

Clients are told about the benefits of a small, wellspaced family, and the problems presented in a large, closely spaced family. They are taught ways which can assist them to space or to stop childbearing. They are told about modern contraceptive methods, their names, what they look like, how to use them, and where to find them. Based on this information, and perhaps on past experience, the client selects the method that she prefers. Based on the method chosen, the nurse examines the client to ascertain the suitability of the method. If on examination the method proves unsuitable for the client, the nurse then advises on the most likely suitable method.

\section{Knowledge and Source of Modern Contraceptive}

The term "family planning" was familiar to all the participants in the study area. But more specific knowledge of the names of the various methods and willingness to talk freely about them differed between regions. In Chogoria, most participants knew the names of at least three family planning methods and could talk freely about them. In Maua, most participants knew only one method of family planning and spoke little about it. The most commonly mentioned methods were the pill, injection, and the IUD.

The most popular source of family planning information was the hospital. A few mentioned volunteer health workers, the church, the chief's baraza, or seminars. The chief's baraza and seminars were mentioned by men from Chogoria, but most participants were women who learned about family planning during clinic visits. However, a majority of the participants did not know how the methods work in their bodies to prevent pregnancy. A woman asked whether she would get pregnant if she took her pill after intercourse. Another one abstained while taking the pill. Yet another used the pill despite having had a tubal ligation. Pictures were presented to one group in order to illustrate how the pill and IUD prevents a pregnancy. The participants were eager to learn and expressed gratitude at the end of the session.

The above knowledge of family planning can be said to be shallow and inadequate. There is thus a need to explain the advantages and shortcomings of the various methods and how they work to prevent pregnancy, so that the clients can be more comfortable with them.

\section{Desired Family Size and Child Spacing}

Most women preferred a family size of between two and four children. This is consistent with an earlier finding that four is the desired family size among fecund women of Chogoria (Goldberg, I.H. et al., I989:2I-22). The participants in this study were between 20 and 40 years of age; the mean family size was 3.2 in Chogoria and 3.7 in Maua. National figures for this age group have been reported as 4.4 (Kenya Census, 1979:68), 4.7 (Kenya, KCPS, 1984:45), and 4.2 (Kenya, KDHS, 1989:24). The figures are not directly comparable, but they do indicate lower completed fertility in Chogoria and Maua than in the entire country for women in this age group. It should be observed that some of these women will probably have more children, but it is also possible that some women will use contraception effectively and realise their desired goal. 


\section{Use of Modern Contraceptives}

After accepting and practicing a modern method of family planning, a client returns for an appointment at which her experience with the method is reviewed. In this review those who are happy with their methods are encouraged to continue using, while those with problems are examined for side effects. Those with serious side effects are advised on alternative methods, and those with minor difficulties are counselled and reassured. Those who fail to attend the appointment are followed up in Chogoria, but not in Maua. The purpose of the follow-up is both to establish why the client failed to attend and to find out how they are progressing with their methods.

Many participants in this study were using family planning. 78 of the participants from Chogoria and 33 percent of those from Maua were currently using a modern method of contraception. The participants from Chogoria unanimously agreed that the decision to use family planning is taken by both the husband and the wife, while in Maua a majority said that the decision is unilaterally taken by either the husband or the wife. An example of a unilateral decision by the wife was a woman from Kyengu who travels for family planning to Maua, a distance of $8 \mathrm{~km}$ from her home. A mobile clinic near her home provides such services, but she prefers to use Maua in order to conceal her use of family planning from her husband.

\section{Satisfaction of Providers and Clients}

All the departments and staff of Chogoria work in unison. For example, clinic officers in the outpatient section assist in distributing condoms to male clients. But at Maua, except for the senior staff, CHD personnel seem to be working in isolation. One CHD nurse from Maua commented with regret, "it is ridiculous that we go to teach the people away from the hospital while our own colleagues like the nurses in the wards do not know what we do in this department."

Chogoria staff was happy with their seminars and workshops. I attended two closing sessions of workshops to train volunteers. In one session a well-performed play portrayed the role of volunteer field workers and nurses in the community. On the other hand, Maua staff were dissatisfied; they complained, for example, about a lack of food for their camping workshops, saying that they depend on the public to provide them with food.

Although staff morale seemed to be higher in Chogoria than in Maua, all were very welcoming and good to work with.

No direct question was asked with regard to client satisfaction with family planning methods. However, indirect inferences can be drawn from the fears clients expressed about various contraceptives. In each area, some women were worried that they might become permanently sterile after injection with Depo-Provera. This apprehension was compounded by the fear that should the woman become sterile, the husband might marry another wife to bear children for him. Others were concerned about their health. For example, they associated cancer with the pill and stomach pains with tubal ligation. Others were anxious that the IUD could cause frequent menstruation or disappear in the body altogether. Yet others did not trust the pill or IUD. Such anxieties and mistrust call for more intensified counselling and more detailed explanations of the how the methods work to avert pregnancy. Better staff training would be required for such specialised tasks.

\section{DISCUSSION}

The family planning programmes of Chogoria and Maua are comparable in many respects. This discussion will highlight the differences between them and the ways the respective programmes might be improved.

Family planning personnel consists largely of nurses, health educators, volunteers, and traditional birth attendants. Although the composition of the family planning personnel is similar, the level of knowledge and the satisfaction with the respective establishment were different. Chogoria staff were more knowledgeable about contraceptives and were more satisfied with the training provided by their hospital than their counterparts at Maua. The relationship between the senior and junior staff was cordial at Chogoria 
but distant at Maua. The hospital staff from Chogoria knew all the field staff by name, but this was not the case at Maua. This close relationship between different grades of staff at Chogoria enabled the junior staff to build confidence in their work. This was apparent as the field educators and volunteers taught the women attending antenatal and postnatal clinics.

The content of the information about contraceptives provided to women attending clinics was similar in both hospitals. But the teachers at Chogoria were more knowledgeable and confident than those at Maua. Neither provided sufficient detail on how the methods work in the body to prevent conception or on the various associated side effects. Such details are necessary to assuage fears women may have about contraceptives. One group of women who were taught the details of how the pill and IUD work were enthusiastic, happy, and grateful to have learned how their bodies function and how contraception works.

Knowledge of at least one method of contraception was widespread but was limited to the name of the method and did not include how it works. With this shallow knowledge some women who were determined to prevent a pregnancy were simultaneously using two relatively effective methods. Such misuse of resources can be alleviated by increasing both the quantity and quality of the information given.

The main sources of family planning knowledge and services were the hospital, the dispensary, or the health centre. There were many health educators and volunteers, but these were not reported as the main source of knowledge since most of the women learn about modern family planning when they attend clinics. According to my observations, women came to the clinics largely for ante-natal and post-natal services and only coincidentally learned about family planning. This implies that the demand for the knowledge of family planning is not well established. Perhaps family planning information should be disseminated in additional places frequented primarily for other services, such as churches and schools.
Many participants in this study were using modern contraception -- 78 and 33 percent in Chogoria and Maua respectively. These figures may not accurately reflect the true levels because the sample size was small and unrepresentative. Nevertheless, they show in relative terms that the prevalence of contraceptive use among the participants in this study was more than twice as high in Chogoria as in Maua. This can be attributed in part to the higher quality of family planning at Chogoria. At Chogoria the seminars and workshops to train health educators and volunteers are well organised and satisfying to the attendants; working morale across various ranks is good; and the teachers of family planning are happy and confident in their work. If a client fails to attend an appointment at Chogoria, a followup is made. At Maua, however, health workers' morale is generally low and defaulters are not followed up.

\section{CONCLUSIONS}

Although many factors affect the uptake of contraceptives, quality of care in family planning, as illustrated in this study, contributes positively towards better knowledge and greater use of modern contraception.

Three conclusions flow from this comparative study. First, the satisfaction of family planning providers and their clients contributes positively towards knowledge and use of modern contraceptives. Second, women feel secure and comfortable with the methods they use when their husbands participate in deciding what methods to adopt. Finally, follow-up services for those who fail to attend appointments help to build rapport between providers and clients and provide an opportunity to learn of the circumstances that lead to discontinuing the use of contraceptives.

\section{RECOMMENDATIONS}

The following recommendations are pertinent to the provision of family planning information and services in the areas studied: Maua should seek to improve interpersonal relationship among the various members of staff and their clients. Maua should seek to involve men in family planning and should follow up on clients who default. Both 
Chogoria and Maua should provide details of how the various contraceptives work to avert conception.

\section{REFERENCES}

- Bauni E.K. (1990) Fertility Decline and Family Planning in Chogoria, Eastern Kenya. Unpublished PhD Thesis. London School of Hygiene and Tropical Medicine, University of London.

- Bruce J. (1990) "Fundamental Elements of the Quality of Care: A Simple Framework". Studies in Family Planning vol.2I, No. 2:61-9I.

- Ferguson A. et al (1988) Family Planning Needs in Colleges of Education. Report of a Study of 20 Colleges in Kenya. Ministry of Health, Division of Family Health, GTZ Support Unit, Nairobi.

- Goldberg I.H. et al ( 1989) "Contraceptive Use and Fertility Decline in Chogoria, Kenya". Studies in Family Planning vol.20, No. I: 17-25.

- Kenya Population Census (1979) 1979 Population Census vol.ll. Analytical Report. Central Bureau of Statistics, Ministry of Finance and Planning, Nairobi, Kenya.

- Kenya, KCPS (1984) Kenya Contraceptive Prevalence Survey 1984. First Report. Central Bureau of Statistics, Ministry of Planning and National Development, Nairobi.

- Kenya, KDHS (1989) Kenya Demographic and Health Survey 1989. National Council For Population and Development, Ministry of Home Affairs and National Heritage, Nairobi. 